\title{
Genome Sequences of Brucella Melitensis Strains QH2019001 and QH2019005 Provides Insight Into Different Evolutionary Origins Within Qinghai Province, China
}

\section{Zhijun Zhao}

Qinghai institute for endemic disease prevention and control

Jiquan Li

qinghai institute for endemic disease preventionand control

\section{Li Ma}

qinghai institute for endemic disease prevention and control Hongmei Xue

qinghai institute for endemic disease prevention and control

\section{Xuxin Yang}

Qinghai institute for endemic disease prevention and control

\section{Yuanbo Zhao}

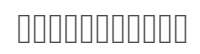

\section{Yumin Qin}

qinghai institute for endemic disease

Xiaowen Yang

China CDC

\section{Dongri Piao}

China CDC

\section{Hongyan Zhao}

Centers for Disease Control and Prevention

\section{Guozhong Tian}

China cdc

\section{Qiang Li}

qinghai institute for endemic disease prevention and control

\section{Guang Tian}

qinghai institute for endemic disease

Jianling Wang

qinghai institute for endemic disease

Hai Jiang

qinghai institute for endemic disease prevention and control 
Liqing Xu ( $\nabla$ qhxlq2006@163.com )

\section{Research Article}

Keywords: Brucella melitensis, WGS, SNP

Posted Date: July 9th, 2020

DOI: https://doi.org/10.21203/rs.3.rs-36867/v1

License: (c) (1) This work is licensed under a Creative Commons Attribution 4.0 International License. Read Full License 


\section{Abstract \\ Background}

Brucellosis, which is cause by Brucella, is one of the most important zoonoses and is considered a "forgotten, neglected zoonosis" by the WHO.

\section{Methods}

Blood samples were isolated from two brucellosis males that were confirmed B. melitensis positive following multiple loci variable-number tandem repeat analysis (MLVA) and strains QH2019001 and QH2019005 were identified. Genomic DNA was extracted from these samples and whole genome sequencing (WGS) was performed. Next, SNP-based phylogenetic analysis was performed to compare the two strains to $94 \mathrm{~B}$. melitensis strains (complete genome and draft genome) retrieved from online databases.

\section{Results}

The QH2019001 and QH2019005 strains were found to have differences in their variable-number tandem repeats. Phylogenetic examination of the 96 strains assigned them to 5 genotype groups, with QH2019001 and QH2019005 assigned to the same group, but to different subgroups; with the QH2019005 strain assigned to a new subgenotype, Ilj. These findings were then combined to determine strain origin.

\section{Conclusions}

Utilizing a whole genome SNP-based approach highlights the diversity between $B$. melitensis strains, and it can facilitate the elucidation of different evolutionary histories. This approach also revealed that the QH2019005 is part of a new subgenotype with an ancient origin in the eastern Mediterranean Sea.

\section{Background}

Brucellosis, which is caused by bacteria in the Brucellagenus, is one of the most important zoonoses worldwide and is considered a "forgotten, neglected zoonosis" by the WHO (World Health Organization 2014) [1]. Brucellosisis endemic in regions within Africa, Asia, and Latin America and other countries along the Mediterranean Sea [2,3].Human infections can occur due to the consumption of contaminated non-pasteurized milk or cheese, or by occupational exposure to infected animals or their carcasses, uterine secretions, or aborted fetuses [4]. While the mortality rate of brucellosis is low, the morbidity rate is much higher. Worldwide, incidence of human brucellosis varies widely, from $<0.01$ to $>200$ per 100,000 people in endemic disease areas [5]. In mainland China, the total incidence rate of human brucellosis 
increased from 0.92per 100,000 people in 2004 to 4.2 per 100,000 people in 2014 [6]. Thus, brucellosis is becoming a major public health problem that impacts human physical and mental health.

Brucella is a gram-negative, facultative, intracellular pathogen that induces cell toxicity by altering the plasma membrane and inducing cell apoptosis [7]. Currently, the Brucella genus contains 11 accepted species, with 6 of those, namely, B. melitensis, B. abortus, B. suis, B. canis, B. ovis, and B. neotomae, considered 'classical' species [8]. While various species differ in host preference, virulence, and/or zoonotic potential, all Brucella species maintain a 97-99\% genomic sequence identity [9].

To ensure accurate epidemiological surveillance and to enable differentiation between infected and vaccinated individuals, species identification and subtyping is essential [10].Currently,Brucella identification and subtyping is performed usingmultiple loci variable-number tandem repeat analysis (MLVA), a genotyping tool for species identification and genetic diversity assessment [11]. However, MLVA is time-consuming and laborious and cannot cover all of the currently known Brucella speciesand biovars. An alternative to the MLVA approach is to utilize whole genome sequencing (WGS)-based strain typing combined with single nucleotide polymorphism (SNP) analysis to obtain a higher resolution and fine-scale differentiation of Brucella genotypes and subgenotypes [12].

In humans, $B$. melitensis, which contains three biovars (1,2 and 3), is the most virulent species and was the predominant strain associated with human brucellosis in China from 1953 to 2013 [13]. While all three biovars cause disease in small ruminants, their geographic distributions vary [14]. In a study examining Brucella in the Qinghai-Tibet Plateau region, none of the genotypes matched any of the sequences in the Brucella 2012 MLVA database, possibly due to these strains having unique geographical characteristics in the Qinghai-Tibet Plateau region and B. melitensis being the predominant species in the area [15].

Recently, the prevalence of human brucellosis in Qinghai Province has increased from 0.04 per 100,000 people in 2011 to 1.96 per 100,000 people in 2018. Cases are distributed across 31 counties within the entire province, thus making infectious source tracking difficult. Herein, two brucellosis positive individuals from the Qinghai-Tibet Plateau region in China were examined and $B$. melitensis strains QH2019001 and QH2019005 were identified using MLVA. These strains were then further examined using WGS and SNP-based phylogenetic analysis to examine associations with other $B$. melitensis strains and to determine origin.

\section{Methods}

\section{Ethics statement}

This research was conducted according to the principles of the Declaration of Helsinki, and the study protocol was approved by the Ethics Committees of the National Institute for Communicable Disease Control and Prevention (Beijing, China). Informed consent was obtained from the two patients who received a brucellosisdiagnosis at the Qinghai Institute for Endemic Disease Prevention and Control 
based on the Diagnosis for Brucellosis Standards (WS 269-2019) used in China. Obtained patient history/data were anonymized for the purpose of this study. Blood samples were obtained and Brucella strains were isolated.

\section{Bacterial strain isolation and MLVA assay}

Blood cultures were examined for brucellosis patients diagnosed at the Qinghai Institute for Endemic Disease Prevention and Control in Qinghai Province, China. Brucella-positive samples were identified as B. melitensis strains QH2019001 and QH2019005 following MLVA as previously described [16]. Briefly, 16 gene loci were examined and divided into 3 groups as follows: panel 1 with 8 loci (bruce06, bruce08, bruce11, bruce12, bruce42, bruce43, bruce45, and bruce55), panel 2A with 3 loci (bruce18, bruce19, and bruce21), and panel 2B with 5 loci (bruce04, bruce07, bruce09, bruce16, and bruce30).

\section{Genomic DNA sample preparation}

Genomic DNA was extracted using a sodium dodecylsulfate (SDS)-based method [17]. The obtained DNA was assessed via agarose gel electrophoresis and quantified using a Qubit 2.0 Fluorometer (Thermo Scientific, USA).

\section{Whole genome sequencing}

Sequencing libraries were generated using a NEBNext® Ultra ${ }^{\text {TM }}$ DNA Library Prep Kit for Illumina (NEB, USA) following the manufacturer's recommendations, with $1 \mu \mathrm{g}$ DNA used per sample. Unique codes were added to attribute sequences to each sample. Briefly, DNA samples were fragmented by sonication to a size of $350 \mathrm{bp}$. The DNA fragments were then end-polished, A-tailed, and ligated with full-length adaptors for Illumina sequencing prior to PCR amplification. Finally, PCR products were purified using an AMPure XP system (Beckman Coulter, USA) and libraries were analyzed for size distribution using an Agilent 2100 Bioanalyzer and quantified using real-time PCR. WGS of the QH2019001 and QH2019005 strains was performed using an Illumina NovaSeq 6000 (PE 150 bp) at Novogene Bioinformatics Technology Co., Ltd. (Beijing, China).

\section{Genome assembly and annotation}

The raw sequencing data were filtered to obtain clean data(the original data must be filtered to obtain valid data).Genome assembly was then performed using the clean data with multiple k-mers as follows:1) assembly with SOAPdenovo using multiple k-mers (default parameters of 95, 107, and 119), with the optimal k-mer obtained by adjusting other parameters $(-\mathrm{d}-\mathrm{u}-\mathrm{R}$, and $-\mathrm{F})$ and by selecting a preliminary assembly with the least scaffolds; 2 ) assembly with SPAdes using multiple k-mers (default was 99 and 127), with the assembly with the optimal k-mer and the least scaffolds selected; 3) assembly with Abyss using a k-mer of $64 ; 4)$ assembly results from the three software were integrated using CISA software and the assembly result with the least scaffolds was selected; 5) Gapcloser was used to fill gaps and filter reads with low sequencing depth ( $<0.35$ of the average depth) to obtain the final assembly result; and 6) fragments below 500 bp were filtered out and the final result was utilized for the 
gene prediction. A whole genome Blast search (E-value less than $1 \mathrm{e}-5$, minimal alignment length percentage larger than $40 \%$ ) was performed with a threshold of identity $\geq 40 \%$ and coverage $\geq 40 \%$. The obtained genomic sequences were deposited in the Microbial Pathogens Database (http://data.mypathogen.org.; accession numbers: ICDC-20200117-135823-300 and ICDC-20200117140311-357).

\section{SNP discovery and phylogenetic construction}

Genomic alignment between the sample genome and reference genome (or among more than two sample genomes) were performed using the MUMmer [18] and LASTZ[19][20] tools. Genomic synteny was analyzed based on the alignment results.

SNP-based phylogenetic analysis was then performed to infer the relationships between the B. melitensis strains. SNP was found by the genomic align ment results among samples by the MUMmer and LASTZ . The phylogenetic trees were constructed using TreeBeST and PhyML with orthologous genes and bootstraps of 1,000 .

For the two strains that were sequenced, rapid whole genome alignment was performed using MUMmer and LASTZ, with 94 strains of B. melitensis that are publicly available utilized as reference genomes. The obtained alignment data were then analyzed for genomic synteny.Gene family was constructed by the gene of the two strains and reference strains, using the multiple softwares: Blast[21] was used to pairwise align all genes and eliminate the redundancy by solar and carried out gene family clustering treatment based on the alignment results with Hcluster_sg software.

\section{Results}

\section{General conditions}

The first B. melitensis strain, QH2019001 (isloated and assayed in 2018, sequenced in 2019 ) was isolated from a male worker in Qinghai province, China. This man went to his hometown (Anhui Province) in April 10, 2019 for 12 days where he had contact with sheep (unknown origin) when cleaning sheepfolds. The second B. melitensis strain, QH2019005, was isolated from a male farmer in Qinghai Province that made a living as a sheep herdsman in Yushu, Maduo, and Haiyan Counties in Qinghai Province. The two males showed clinic symptoms and had positive serum agglutination tests (SATs) at titers of 1:100. When examining epidemic factors, clinical manifestation and serum detection (Table 1), the two males were diagnosed as brucellosis positive and the blood cultures resulted in the isolation of Brucella strains QH2019001 andQH2019001 that were identified asB. melitensis biovar 3.

\section{Table1.General brucellosis patient information.}




\begin{tabular}{|llllll|}
\hline StrainIDs & Host & Sex & Age & Occupation & Symptoms \\
\hline QH2019001 & human & male & 37 & worker & intermittentfever \\
\hline QH2019005 & human & male & 34 & farmer & \\
& & & & & headache,weak,sweating, \\
& & & & & backpain,\&persistentfever \\
\hline
\end{tabular}

A MLVA-16 assay was used to determine the genotypes for $B$. melitensis strains QH2019001 (1- 4 - 3 - 13 2 - 2 - 3 - 2 - 4 - 40 - 8 - 6 - 4 - 3 - 18 - 5) and QH2019005 (1 - 5 - 3 - 13 - 3 - 2 - 3 - 2 - 4 - 38 - 8 - 5 - 4 - 7 - 16 5). The two strains showed a $99.1 \%$ genotype similarity. For the two $B$. melitensis strains, variablenumber tandem repeat (VNTR) differences were present in bruce08 and bruce42 (panel 1); while, bruce19, bruce21, bruce 04, bruce09, and bruce16 (panels 2A and 2B) also exhibited VNTR differences.Furthermore, thetwo B. melitensis strains were also similar to the other strains in Qinghai Province.When combining these findings with epidemical data, B. melitensis strain QH2019001 appears to have been imported from Anhui Province, while the QH2019005 strain appear to be native to Qinghai Province (Fig. 1).

\section{Genomiccharacteristic}

To determine the origins of the two strains, WGS was performed using a high performance sequencing platform, lon Torrent PGM (Life Technologies, USA), and followed by de novo assembly. The two strains comprised 24 contigs, with N50s 297 and 202 bp (QH2019001) and 332 and 914 bp (QH2019005). The total contig sequence lengths were 3,291,786 bp for QH2019001 and 3,289,996 bp for QH2019005, with both having a GC content of $57.24 \%$.

The obtained sequences were then compared to 94 B. melitensis strain sequences using TreeBeST and PhyML. Phylogenetic analysis based on all of the whole genome SNPs provided insight into the overall geographical distributions of the 96 examined $B$. melitensis strains. The strains were divided into five major genotypes as follows: genotype I, Bruc048 and its related strains; genotype II, UK3/06 and its related strains; genotype III, strains of African origin; genotype IV,B115 and its related strains; andgenotype V, $16 \mathrm{M}$ and its related strains (Fig. 2). The QH2019001 and QH2019005 strains were all associated with the genotype II group. Furthermore, several clades and subclades were isolated within the lineages and were associated with geographic attributes. The QH2019001 strain was assigned to subgenotype Ilh, while the QH2019005 strain was assigned to subgenotype Ilj (a new subgroup).

\section{Discussion}

Due to thehigh mutation rate in tandem DNA repeats loci, the MLVAplatform provides the ability to distinguish field and vaccine strains [22], associate isolates with geographic origins, and discriminate most Brucella species with a high homogeneity discriminatory power [23]. In China, MLVA genotyping provides a basis for reliable disease monitoring when combined with strain phylogeny and regional and time distributions, thus potentially providing valuable insight when examining an epidemiological linkage 
or trace-back investigation during a brucellosis outbreak [13, 24, 25].Furthermore, previous studies have shown thatMLVA can be used as an epidemiological tool for strain resolution and to distinguish relapses from reinfections in brucellosis patients [26]. The two Brucella strains identified herein, which were isolated from the Qinghai province, had difference when compared to those in the Brucella 2012 MLVA database [1]. Furthermore, the QH2019001 and QH2019005 strains did exhibit VNTR difference, but most of the strains were similar to each other. However, due to the high genetic diversity of the Brucella genus, the MLVA approach can be limited based on the nature of VNTRs and the technique itself [27], thus the exact origins of the two strains could not be determined.

One study showed that when utilizing only a MLVA approach, only 52 genotypes from 63 patients can be distinguished; however, when utilizing a whole genome SNP-based approach, all patients can be distinguished from each other,thus demonstrating a higher discriminatory power [28]. Furthermore, WGSbased analysis has been shown to distinguish very closely related B. melitensis strains (up to 6 genes or 7 SNPs difference), while MLVA profiling might not provide enough resolution to accurately predict phylogenetic relationships [29]. Thus, SNP data can enable the development of novel high-resolution molecular typing techniques that can discriminate inter- and intraspecies relationships in pathogenic microorganisms.

Following phylogenetic clustering, the $B$. melitensis strains $(\mathrm{n}=96)$ were divided into five genotypes [24], with genotype I forming the earliest diverging clade. Most of the Asian isolates of $B$. melitensis clustered into genotype II,while genotypelll was associated with Africaand genotypes IV and V were associated withEurope and the Americas, respectively, which is consistent with previous findings [30].

The genotype II strains had the widest geographical distribution after diverging from genotype I. The diversification of this genotype might have taken place in the second half of the third millennium BC, approximately 5,270 years ago [31]. The most important land routes connecting Eastern Europe with India on the western part of the Silk Road went through Central Asia or the Caucasus and Iranian highlands, thus the Arabs played a key role in Europe's trade with Southeast Asia and China. Moreover, genotype II includes nine previously described subgenotypes (Ila-IIi)[30, 31].

Following WGS, the two strains, QH2019001 and QH2019005, were compared to 94 B. melitensis strains and were both assigned to genotype II, but to different subgroups.Furthermore,B. melitensis strain QH2019005 was assigned to genotype Ilj (a new subtype), which is closely associated with subgenotype Ila that contained only a single strain, UK3/06. The QH2019001 strain was assigned to subgenotype Ilh.The differences between the QH2019001 and QH2019005 strains suggest that the nucleotide variation may be attributed to changes in geographic distribution. It is also possible that subgenotype Ilh diversification may have occurred during the second half of the XIV century A.D. and it may have been driven by therelatively isolated and more unique environment of the Qinghai-Tibet Plateau, with its extremely high altitudes. Due to the environment, it is difficult for lowland livestock breeds or wild animals to survive; hence, livestock exchange between Qinghai Province and other regions would be limited [14]. The QH2019005 strain was the most similar to the UK3/06 strain that was isolated from the Near East, 
thus suggesting that this strain and its new subgenotype have ancient origins. TheQH2019001 strain was the most similar to the I-349 strain that was isolated from Central Asia (Russia: Republic of Tuva) [32], thus suggesting that this strain shares a common origin. Active trade between Russia and China could have promoted the transmission of B. melitensis strain QH2019001 to other regions (Anhui Province) in China (Fig. 3).

\section{Conclusion}

This study utilized whole genome SNPanalysis and identified B. melitensis strains QH2019001 and QH2019005 that were isolated in Qinghai Province. When comparing these strains with 94 database obtained strains, a high degree of diversity was noted. The two strains were found to have different evolutionary histories, with the native strain QH2019005 assigned to a new subgenotype with an ancient origin in the eastern Mediterranean region.Thus, utilizing a whole genome SNP approach can facilitate the examination of $B$. melitensis interspecies relationships in theendemic Qinghai-Tibet Plateau region.

\section{List Of Abbrevation}

whole genome sequencing: WGS

multiple loci variable-number tandem repeat analysis : MLVA

single nucleotide polymorphism : SNP

serum agglutination tests : SAT

variable- number tandem repeat: VNTR

\section{Declarations}

\section{Acknowledgments}

We thank LetPub (www.letpub.com) for its linguistic assistance during the preparation of this manuscript.

\section{Author Contributions}

Conceptualization: Hai Jiang and Liqing Xu

Formal analysis: Zhijun Zhao and Jiquan Li

Investigation:Zhijun Zhao, Jiquan Li, Li Ma, Hongmei Xue, Xuxin Yang,Yuanbo Zhao, Yumin Qin, Guang Tian and Qiang Li

Methodology: Dongri Piao, Hongyan Zhao, Guozhong Tian and Xiaowen Yang 
Project administration: Hai Jiang and Liqing Xu

Supervision:Liqing $\mathrm{Xu}$

Writing-original draft: Zhijun Zhao and Jiquan Li

Writing-review \& editing: Hai Jiang

\section{Funding}

This study was funded by the National Science and Technology project "Major Infectious Diseases such as AIDS and Viral Hepatitis Prevention and Treatment" (2018ZX10712-001) in China and by the National Natural Scientific Foundation of China (30972556).

\section{Availability of data and materials}

All of the relevant data are within the manuscript and its supporting information files.

\section{Ethics approval and consent to participate}

This research was conducted according to the principles of the Declaration of Helsinki, and the study protocol was approved by the Ethics Committees of the National Institute for Communicable Disease Control and Prevention (Beijing, China). Informed consent was obtained from the two patients who received a brucellosis diagnosis at the Qinghai Institute for Endemic Disease Prevention and Control based on the Diagnosis for Brucellosis Standards (WS 269-2019) used in China. Obtained patient history/data were anonymized for the purpose of this study. Blood samples were obtained and Brucella strains were isolated.

\section{Consent for publication}

Not applicable.

\section{Competing interests}

The authors declare that they have no competing interests.

\section{Author details}

1. Qinghai Institute for Endemic Disease Prevention and Control, Xining 811602, Qinghai, China

2. State Key Laboratory for Infectious Disease Prevention and Control, Collaborative Innovation Center for Diagnosis and Treatment of Infectious Diseases, National Institute for Communicable Disease Control and Prevention, Chinese Center for Disease Control and Prevention, Beijing, China

\section{References}


1. World Health Organization. 2014. The control of neglected zoonotic diseases. In: NZD4 organising committee, editor. WHO conference report. Geneva: WHO Press. pp. 23-35.

2.Felipe F T, Regina B G, Natacha C. Human-to-human transmission of Brucella - a systematic review. Tropical Medicine and International Health , 2017®22:539-546.doi: 10.1111/tmi.12856

3.Rubin B, Band JD, Wong P, et al. Person-to-person transmission of Brucella melitensis. Lancet, 1991 ; 1:14-15. doi: 10.1155/1995/909404.PMCID: PMC3327908

4. Alcina V C N, Juliana PS M, Mariana N X, Tatiane A, Andrey P L , Renato L S. Pathogenesis of bovine brucellosis. The Veterinary Journal, 2010囚 184:146-155.

5. Hanot MD , Boarbi S, Michel P, et al. Imported human brucellosis in Belgium: bio and molecular typing of bacterial isolates, 1996-2015. Plos one. 2017;12:e0174756.

6.S Lai, H Zhou, W Xiong e, t al. Changing epidemiology of human brucellosis, China , 1955-2014. Emerging Infectious Diseases,2017囚 23:184-194

7. Garrity, G.M . 2001. Bergey's Manual of Systematic Bacteriology, second ed. Springer, New York. 721 pp.

8. Gamal W, Falk M, Herbert T, Uwe R, Heinrich N. Detection of Brucella abortus DNA in aborted goats and sheep in Egypt by real-time PCR. BMC Res Notes ,2015囚8:212. Doi: 10.1186/s13104-015-1173-1

9『Marcela SE, Kate S. B, Nazareth RV, Gabriela HM,Elıas BC, et al. Brucella genetic variability in wildlife marine mammals populations relates to host preference and ocean distribution. Genome Biol. Evol.2017囚 9:1901-1912. doi:10.1093/gbe/evx137

10.Hai J, Pengcheng D, Wen Z, Heng W, Hongyan Z, Dongri P囚et al .Comparative genomic analysis of Brucella melitensis vaccine strain M5 provides insights into virulence attenuation .Plos one ,2013,8:e70852

11.A. C. Ferreira, R. Dias, M. I. C. de S'a, R. Tenreiro. Whole genome mapping reveals a large chromosomal inversion on Iberian Brucella suis biovar 2 strains. Veterinary Microbiology, 2016ه192:220-225

12. Sankarasubramanian J, Vishnu US, Sridhar J, Gunasekaran P, Rajendhran J. A genome-wide SNP based phylogenetic analysis distinguishes different biovars of Brucella suis. Infect Genet Evol 2016; 41: 213-17.

13. Guo-Zhong T, Bu-Yun C, Dong-Ri P, Hong-Yan Z, Lan-Yu L, Xi L,et al .Multi-locus variable-number tandem repeat analysis of Chinese Brucella strains isolated from 1953 to 2013. Infectious Diseases of Poverty $\llbracket 2017 \llbracket 6: 89$.doi:10.1186/s40249-017-0296-0 
14.Sofia C, Evridiki B,Antonios Z, Eleni M, Vassilios S, Anna P ,et al . Novel diagnostic approach on the identification of Brucella melitensis Greek endemic strains-discrimination from the vaccine strain Rev. 1 by PCR-RFLP assay. Veterinary Medicine and Science ,2018 4:172-182

15.JunYing M, Hu W, XueFei Z, LiQing X, GuiYing H, Hai J, et al .MLVA and MLST typing of Brucella from Qinghai, China. Infectious Diseases of Poverty 2016ه4: 1-8.doi:10.1186/s40249-016-0123-Z

16.Le FP, Jacques I, Grayon M, Al DS, Bouchon P, Denoeud F, Nockler K, Neubauer H, Guilloteau LA, Vergnaud G: Evaluation and selection of tandem repeat loci for a Brucella MLVA typing assay. BMC Microbiol 2006ख6:9.

17.Lim H J , Lee E.-H, Yoon Y , et al. Portable lysis apparatus for rapid single-step DNA extraction of Bacillus subtilis. J Applied Microbiology, 2016区120(2):379-387

18区Arris R S. Improved pairwise alignment of genomic DNA[M]. ProQuest, 2007.

19凶Chiaromonte F, Yap V B, Miller W. Scoring pairwise genomic sequence alignments[C]/ /Pacific Symposium on Biocomputing. 2001, 7: 115.

20区Altschul S F, Gish W, Miller W, et al. Basic local alignment search tool[J]. Journal of molecular biology, 1990, 215(3): 403-410.

21هKrzywinski, M. et al. Circos: an Information Aesthetic for Comparative Genomics. Genome Res (2009) 19:1639-164

22 Shevtsova E, Shevtsov A, Mukanov K, Filipenko M, Kamalova D, Sytnik I, et al. Epidemiology of

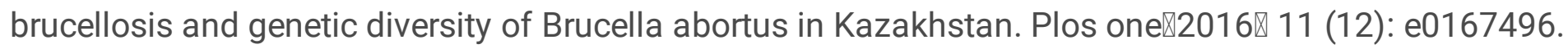
doi:10.1371/journal. pone.0167496

23 Zhi-guo L, Li-jun W, Dong-ri P, Miao W, Ri-hong L, Hong-yan Z, et al. Molecular investigation of the transmission pattern of Brucella suis 3 from Inner mongolia, China. Frontiers in Veterinary Science, 2018》 $5: 271$

24.Fengbo Z, Zhiwei L, Xiaolin L, Xiumin M, Yaoxin Z, Ping J, et al. Multiple-locus variable-number tandem-repeat analysis of Brucella isolates from patients in Xinjiang China. Int J Clin Exp Med》 2015;8(9):15716-15723

25.Hai J, Heng W, Liqing X, Guiying $H$, Junying $M$, et al. MLVA genotyping of Brucella melitensis and Brucella abortus isolates from different animal species and humans and identification of Brucella suis vaccine strain S2 from cattle in China. Plos one,2013®8: e76332

26 Mireille M. Kattar,Rola F. Jaafar, George F. Araj, Philippe Le Fle`che, Ghassan M. Matar, et al .Evaluation of a multilocus variable-number tandem-repeat analysis scheme for typing human Brucella isolates in a 
region of brucellosis endemicity .J Clinical Microbiology 2008₫46( 12):3935-

3940.doi:10.1128/JCM.00464-08

27.Garofolo G, Ancora M, Di Giannatale E. MLVA-16 loci panel on Brucella spp. using multiplex PCR and multicolor capillary electrophoresis. J Microbiol Methods $₫ 2013 \otimes 92: 103-107$.

https://doi.org/10.1016/j.mimet .2012.11.007.

28.Enrico G, Mathias CW, Marie-Theres P, Bernd HN , Lesca M, et al. Whole genome sequencing of Brucella melitensis isolated from 57 patients in Germany reveals high diversity in strains from Middle East. PLoS ONE ,2017ه12(4): e0175425.doi: 10.1371/journal.pone.0175425.PMCID: PMC5384748

29. Anna Janowicz, Fabrizio De Massis, Massimo Ancora, Cesare Cammà, Claudio Patavino,et al .Core Genome multilocus sequence typing and single nucleotide polymorphism analysis in the epidemiology of Brucella melitensis infections. Journal of Clinical Microbiology, 2018》 56 『9『: e00517-18

30.Sarwar Azam,Sashi Bhushan Rao,Padmaja Jakka, Veera NarasimhaRao,Bindu Bhargavi, et al .Genetic characterization and comparative genome analysis of Brucella melitensis Isolates from India. International Journal of Genomics,2016هArticle ID 3034756, 13 pages .doi: 10.1155/2016/3034756 .PMCID: PMC4976149

31.Sergey V. P, Dmitry A. K, Anna S. V , Dmitry G. P, Diana V. R, et al .Global evolution and phylogeography of Brucella melitensis strains. BMC Genomics $₫ 2018 \rrbracket 19: 353$.doi: 10.1186/s12864-018-4762-2.PMCID: PMC5946514

32. Cynthia C. Encyclopedia of world trade: from ancient times to the present.

Armonk: Sharpe Reference; 2005.

\section{Figures}




\section{Figure 1}

Dendrogram based on MLVA-16 genotyping. This figure displays the relationships between B. melitensis strains in Qinghai province. 


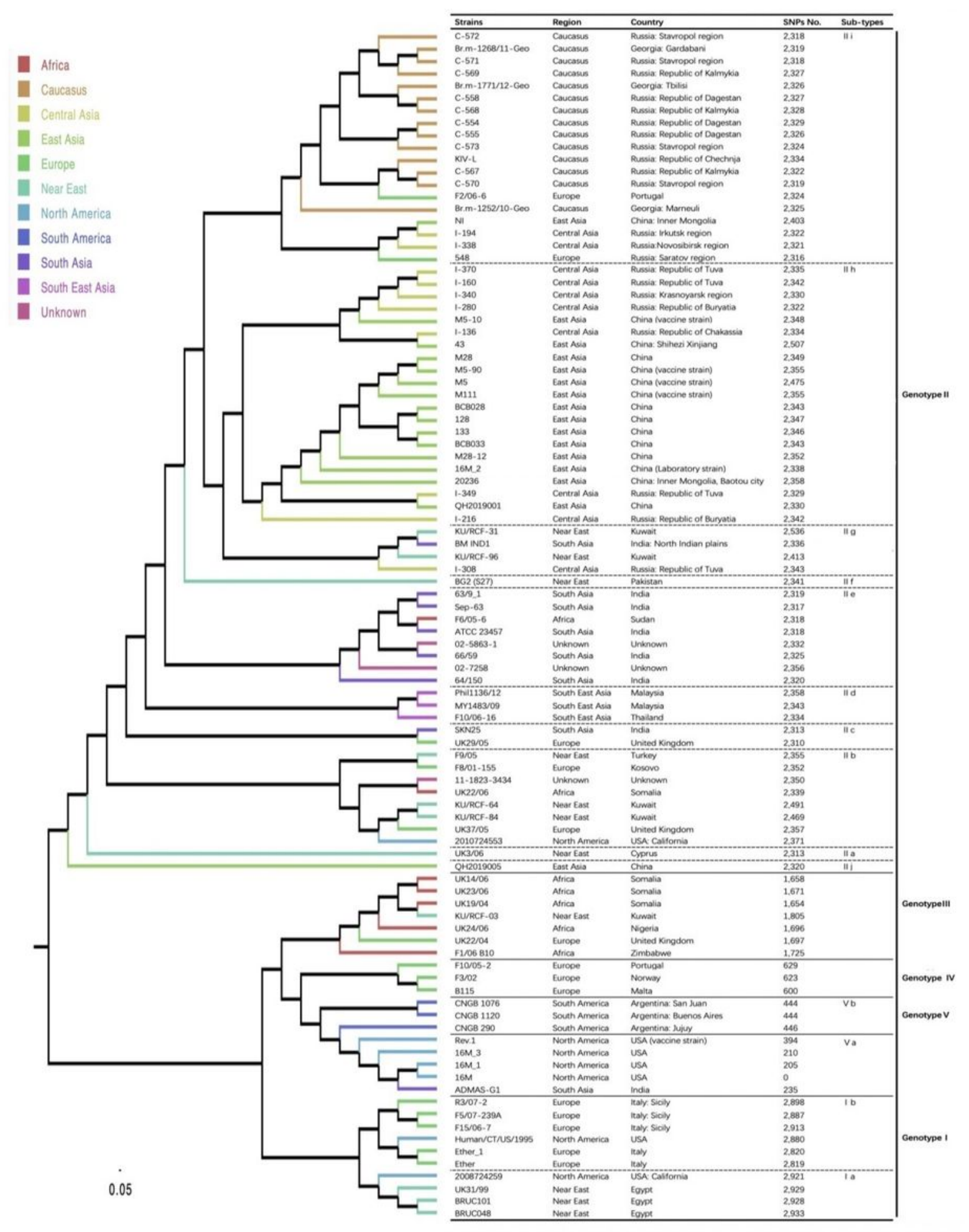

Figure 2

The phylogenetic tree shows the evolutionary relationships of $96 \mathrm{~B}$. melitensis strains. Terminal branch colors correspond to the region where a given strain was isolated. 


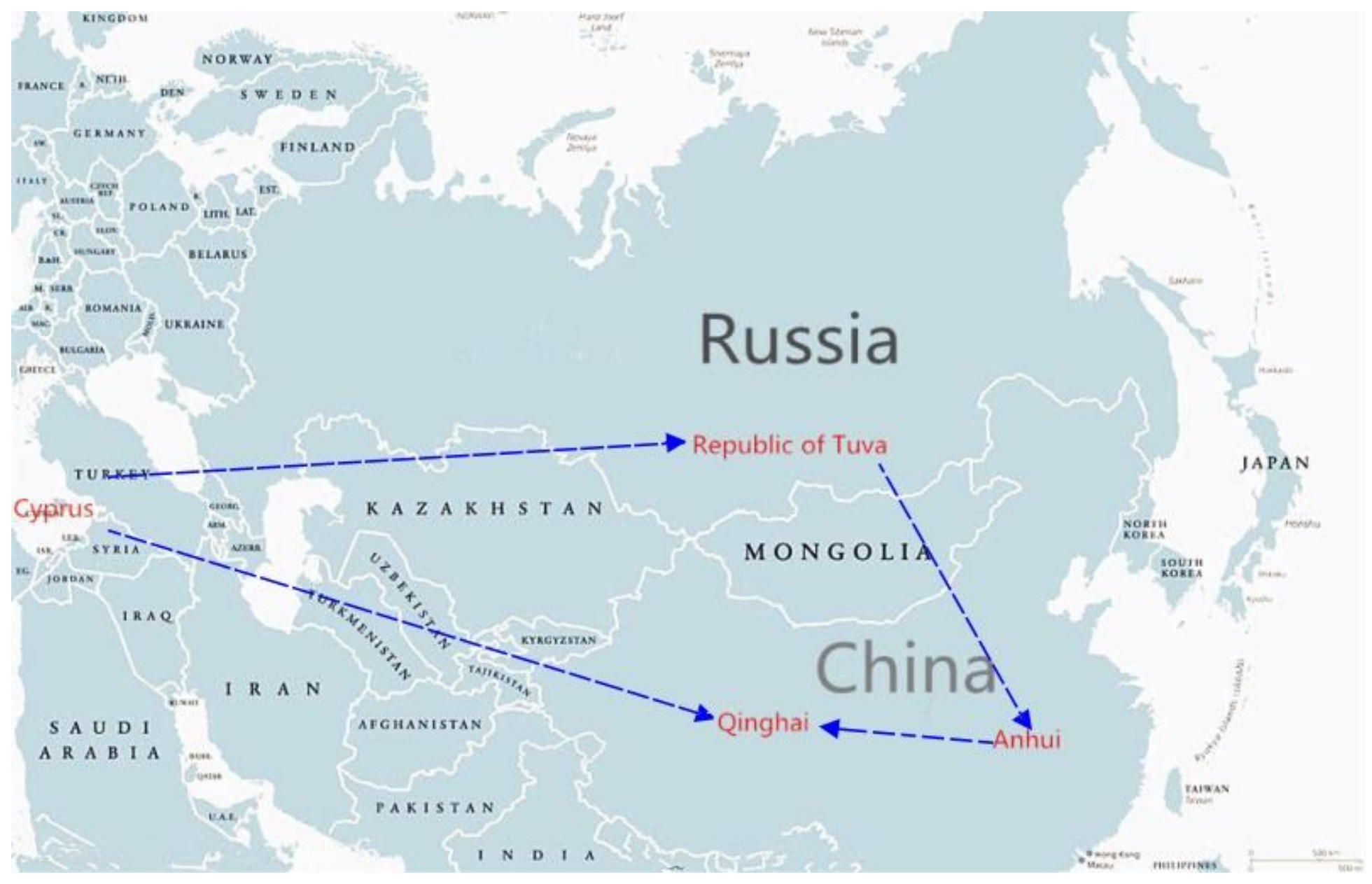

Figure 3

Transmission routes of B. melitensis strains QH2019001 and QH2019005.

\section{Supplementary Files}

This is a list of supplementary files associated with this preprint. Click to download.

- ListofB.melitensisgenomesinformationsupplement.xls 